\title{
Prediction of blood back spatter from a gunshot in bloodstain pattern analysis
}

\author{
P. M. Comiskey, ${ }^{1}$ A. L. Yarin,,${ }^{1,}$ S. Kim, ${ }^{2}$ and D. Attinger ${ }^{2}$ \\ ${ }^{1}$ Department of Mechanical and Industrial Engineering, University of Illinois at Chicago, \\ 842 W Taylor Street, Chicago, Illinois 60607-7022, USA \\ ${ }^{2}$ Department of Mechanical Engineering, Iowa State University, 2529 Union Drive, \\ Ames, Iowa 60011-1210, USA
}

(Received 16 March 2016; published 2 August 2016)

\begin{abstract}
A theoretical model for predicting and interpreting blood-spatter patterns resulting from a gunshot wound is proposed. The physical process generating a backward spatter of blood is linked to the Rayleigh-Taylor instability of blood accelerated toward the surrounding air, allowing the determination of the initial distribution of drop sizes and velocities. Then the motion of many drops in air is considered with governing equations accounting for gravity and air drag. Based on these equations, a numerical solution is obtained. It predicts the atomization process, the trajectories of the back-spatter drops of blood from the wound to the ground, the impact angle, and the impact Weber number on the ground, as well as the distribution and location of bloodstains and their shape and sizes. A parametric study is undertaken to predict patterns of backward blood spatter under realistic conditions corresponding to the experiments conducted in the present work. The results of the model are compared to the experimental data on back spatter generated by a gunshot impacting a blood-impregnated sponge.
\end{abstract}

DOI: 10.1103/PhysRevFluids.1.043201

\section{INTRODUCTION}

A blood-spatter pattern is a collection of bloodstains produced by drops of blood that have traveled through the air, from a source location to a target surface. One of the main issues in forensic analysis of blood-spatter patterns is to determine where a blood spatter originates from. This serves the purpose in crime scene reconstruction of determining the position of the source of blood. This type of knowledge is expected by the analyst communities [1] as it provides a deeper understanding of what occurred during the crime. The position of the source of blood is typically obtained by backward reconstruction of the trajectories, from the target to the source. A sound fluid-mechanical theory of drop atomization, trajectories, and impact would be relevant to the interpretation of blood spatters resulting from gunshot wounds such as the infamous case of Phil Spector, where the question arose of if blood from the back spatter could travel a distance of approximately $1.8 \mathrm{~m}$ [2]. Backward reconstruction of drop trajectories is typically based on the inspection of bloodstains and on a model for the flight of drops [3].

The terminology used for trajectory reconstruction is illustrated in Fig. 1. The three-dimensional region of origin of a blood spatter is called the area of origin and its normal projection onto a horizontal plane is the area of convergence. The area of convergence corresponds to the projection of the trajectories on a horizontal plane. To determine the area of origin, bloodstain pattern analysis (BPA) experts use the angle of impact $\alpha$ from several stains using visual inspection [3]. This angle is the acute angle at which a blood drop impacts the deposition surface, e.g., a floor [see Fig. 1(b)]. Four different angles of impact are denoted by $\alpha_{1-4}$ in Fig. 1(a). As an example, Fig. 1(a) shows the area of origin as the region near the head of the victim, while the area on the floor supporting the feet of the victim is the area of convergence.

\footnotetext{
*Corresponding author: ayarin@uic.edu
} 

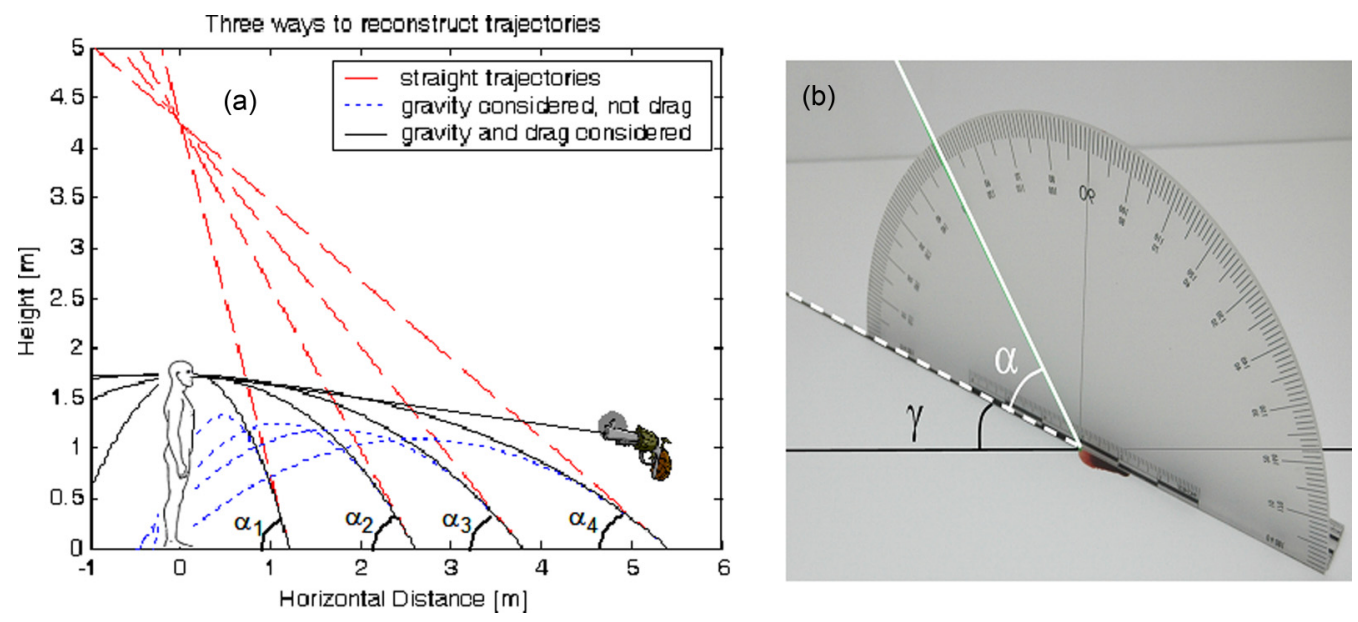

FIG. 1. (a) Reconstruction of the trajectories associated with four bloodstains using straight trajectories versus ballistic trajectories and the geometry (locations of the stains, of the victim, and the impact angles). Panel (a) is reproduced from teaching material graciously provided by $\mathrm{H}$. McDonell. Drop size is assumed to be $3 \mathrm{~mm}$, implying velocities in the 5.3-7.5 m/s range. (b) Definition of the angle of drop impact $\alpha$ and directional (sideways) angle $\gamma$, which is equal to the angle between the vertical plane containing the drop trajectory and another vertical plane containing the bullet trajectory and the target.

As stated in 1939 in Ref. [4], reconstructing trajectories is very difficult and still is today. A first-order approach to reconstruct trajectories is to assume that the drops travel in a straight line [1]. Reconstruction methods based on straight trajectories, such as the method of strings or the trigonometric method, have been used in crime scene reconstruction since at least the 1950s [5] and implemented in software routinely used in crime scenes analysis [6-10]. There is abundant BPA literature on the uncertainties associated with the choice of stains and their inspection to determine the angle of impact and area of origin, as reviewed in Ref. [3]. The actual trajectories are curved, due to the effect of gravity, which is amplified by the air drag. Since gravity and drag act, respectively, vertically and parallel to the trajectory, it is valid to assume that the projection of the trajectories on a horizontal plane consists of straight lines. This justifies the use of straight lines to determine the region of convergence.

However, the use of straight lines to predict the vertical position of the source, i.e., the region of origin, involves a systematic error. For instance, [11,12] report errors in the determination of the region of origin. Similarly, [13] verifies experimentally that the method of strings "[overestimates] the height of the point of origin and the error associated with this technique is significant $(50 \%$ on average)."

Recently, BPA research has proposed methods to reconstruct curved trajectories. Reference [14] describes "a method of reconstructing the area of origin in a nonlinear manner" and incorporates the effects of drag in trajectory calculations using probability densities. A statistical procedure [15] based on aggregate statistics and the basic equations of projectile motion have been shown to determine the area of origin of a blood spatter for cases when the spatter is launched within a narrow range of polar angles. Since ballistic reconstruction involves the knowledge of impact velocity and drop size, a significant amount of recent BPA work has focused on obtaining that information by inspecting the stains, as proposed in Ref. [16]. The authors of [17] reconstructed drop trajectories using ballistic calculations, considering gravity and drag forces, and determined the region of origin with about four times higher accuracy than under the assumption of straight trajectories.

It should be emphasized that none of the existing theories used for predictions of blood trajectories address basic questions such as what the physical mechanism of drop formation in blood spatter is and how it determines the drop size distribution and the initial velocities and angles. Even though a 
drop size can be reconstructed from an available bloodstain, the initial drop velocity and direction cannot be established this way, which adds additional unknowns in any method of prediction of drop trajectory. In fact, the key element of the problem is in these initial conditions rather than in predicting trajectories, albeit the methods of predicting trajectories should also be upgraded to account for the collective effect on the air drag experienced by drops in dense clouds.

Spatter originates from blood at body temperature $\left(\sim 37^{\circ} \mathrm{C}\right)$, while drops typically travel through colder air and impact a colder surface. The issue of the cooling of blood drops in flight and during impact [18] has never been addressed in the context of BPA. This issue might be important because the physical properties of blood vary considerably. When cooled down from $37^{\circ} \mathrm{C}$ to $20^{\circ} \mathrm{C}$, blood viscosity and surface tension increase by $60 \%$ and $27 \%$, respectively. Such significant changes of physical properties affect the Reynolds and Weber numbers, which might affect in-flight atomization, as well as drop spreading and splashing on impact. A related issue is the evaporation of drops during flight, which might be most important for small drops traveling at high velocities because their surface-to-volume ratios are high.

There are four additional obstacles to the ballistic reconstruction of blood drop trajectories. Namely, (i) the area of drop origin can be distinct from the area of origin of blood spatter because the drop generation process can involve a sequence of topological changes (secondary, tertiary, etc., atomization in flight), for example, during the breakup of liquid sheets [19]. (ii) Air through which blood drops fly is not always at rest; sometimes air plays an active role in the drop transport. For instance, experiments involving respiration-caused blood spatter have demonstrated the importance of understanding the role of the surrounding air [20], which helped carrying submillimeter drops further away than occurs in still air. (iii) When spatters have a predominant direction, such as for gunshot back-spatter patterns, the trajectory reconstruction approach for earlier drops requires one to account for the formation flight effect, important in spatters generated from a jet or stream of blood (arterial gushing, expectorate, or gunshot). For instance, in situations when blood spatter is caused by a bullet entering a body, e.g., as in the case of Phil Spector allegedly murdering Lana Clarkson by a gunshot into her open mouth, multiple blood drops issued at a high speed of the order of $10 \mathrm{~m} / \mathrm{s}$ or more, formed a rapidly moving turbulent two-phase jet entraining air. Similar situations are familiar in relation to sprinkler jets, where it was shown that drop motion is dominated by the collective effect, which diminishes the effect of air drag [21,22]. (iv) Blood is a non-Newtonian liquid that is a suspension of red and white blood cells and platelets in lymph. Blood is known to be a shear-thinning fluid that can be described by the power-law model in simple shear flows. Also, viscoelastic properties of blood were revealed experimentally in Ref. [23]. To what extent the non-Newtonian rheological properties of blood affect blood spatter, especially the initial atomization stage, is currently unknown.

In this work a rigorous fluid dynamical model of backward blood-spatter formation as a result of a bullet-inflicted wound is proposed. Also, a fluid dynamical model to predict motion of dense blood drop clouds from the origin to the ground is formulated, implemented, and compared to the experimental data.

\section{EXPERIMENT}

The back-spatter patterns on paper substrate $\left(55.7 \times 70.9 \mathrm{~cm}^{2}\right)$ were created in an indoor shooting range at the Fort Dodge Police Department in Iowa, USA. Swine blood (USDA) with $40 \%$ hematocrit was absorbed in a sponge target $\left(8 \times 4.8 \times 11 \mathrm{~cm}^{3}\right)$, which was suspended on a frame at a known distance from the floor. For each experiment, a single bullet was shot toward the center of the blood-soaked sponge target by a well-trained shooter with a Glock 23 [0.40 caliber, hollow-point bullet (Speer); see Fig. 2(a)]. Relative humidity within the room was $39.0 \%$ and the temperature for both the room and blood was $23^{\circ} \mathrm{C}$. The paper substrate was located on the floor between the shooter and the target to collect back-spatter drops of blood. Two initial bullet inclination angles relative to the horizon were used in the experiments discussed in the present work, namely, $\delta=0^{\circ}$ and $60^{\circ}$ [see Fig. 2(b)]. 
(a)

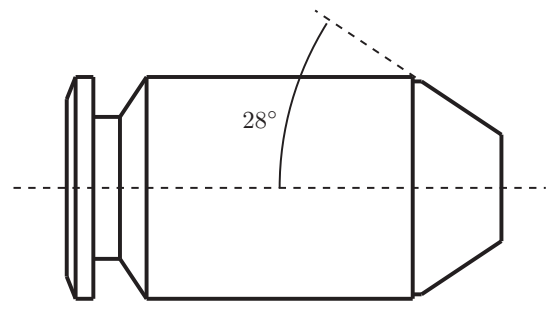

(b)

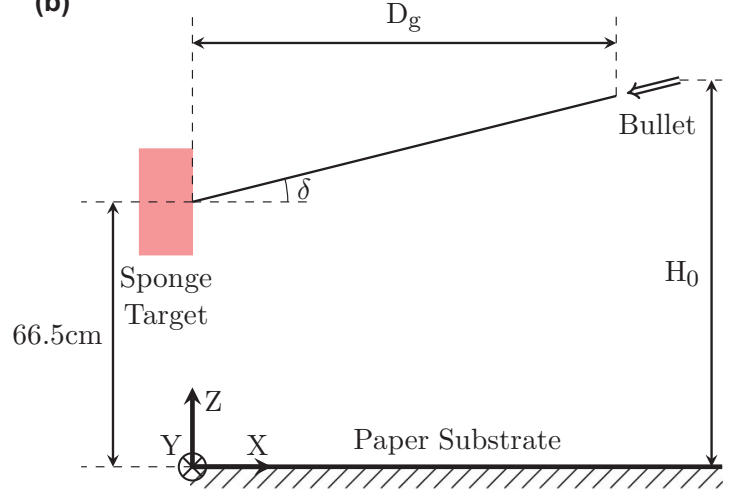

FIG. 2. (a) Geometry of a 0.40 caliber hollow-point bullet. (b) Sketch of the experimental setup with the horizontal paper substrate used to collect back-spatter blood drops. The $Y$ axis directed into the figure and the coordinate trihedron $X Y Z$ is located on the floor under the center of the sponge target, approximately at the location of the bullet impact.

Each test performed was denoted using the following nomenclature: The first position denotes the paper substrate orientation ( $\mathrm{H}$ standing for horizontal), the second position indicates the initial bullet inclination angle (e.g., a0 denotes a zero bullet inclination angle $\delta=0^{\circ}$ ), and the third position describes the horizontal distance from the gun barrel exit to the target in $\mathrm{cm}$. For example, H-a0-D300 stands for a horizontal paper sheet (located on the floor) in the case of the initial bullet inclination angle $\delta=0^{\circ}$ and the distance from the gun barrel exit to the target is $300 \mathrm{~cm}$. Trial numbers for each test case were appended at the end of the experimental nomenclature, e.g., as -\#1 and -\#2. Table I summarizes all the experimental tests with the trial parameters listed.

The blood used in this study was purchased from the National Animal Disease Center, in Ames, IA. The anticoagulant used was $1 \%$ heparin. The hematocrit of the blood was $39 \%$. The room temperature was $23.6^{\circ} \mathrm{C}$. Because blood is a shear-thinning fluid, the determination of viscosity is of interest. As the velocity of the blood resulting from the bullet impact is high, the viscosity at high shear rate $\left(2000 \mathrm{~s}^{-1}\right)$ is to be implied. The viscosity of the blood can be estimated based on the previous viscosity measurement (Table II). From the data in Table II, the viscosity at a shear rate of $2000 \mathrm{~s}^{-1}$ can be expressed as a linear function of temperature and hematocrit using the expression $\mu(2000)=2.9155-0.796 T+0.068 H$. Accordingly, the estimated blood viscosity in this experiment was $3.69 \mathrm{cP}$.

\section{THEORY}

\section{A. Blood back spatter as a result of the Rayleigh-Taylor instability}

Bullet impact phenomena are short-term events taking place on the scale of $3 \mu$ s or even shorter, estimated with the depth of penetration of about $1 \mathrm{~cm}$ and the velocity of about $340 \mathrm{~m} / \mathrm{s}$. In such cases, viscous forces are always negligible compared to the inertial ones, as the Reynolds number is of the order of $10^{7}$. Moreover, in the impact hydrodynamics when pressure is high and flow

TABLE I. Experimental trial parameters; additional nomenclature is defined in Fig. 2(b).

\begin{tabular}{lccc}
\hline \hline Experiment & $\delta(\mathrm{deg})$ & $D_{g}(\mathrm{~cm})$ & $H_{0}(\mathrm{~cm})$ \\
\hline H-a0-D300 & 0 & 300 & 68.6 \\
H-a60-D150 & 60 & 150 & 226 \\
\hline \hline
\end{tabular}


TABLE II. Viscosity measurement at different temperatures and hematocrits.

\begin{tabular}{lccclc}
\hline \hline $\begin{array}{l}\text { Blood } \\
\text { sample No. }\end{array}$ & $\begin{array}{c}\text { Temperature } \\
\left({ }^{\circ} \mathrm{C}\right)\end{array}$ & $\begin{array}{c}\text { Hemotacrit } \\
(\%)\end{array}$ & $\begin{array}{c}\text { Viscosity }(\mathrm{cP}): \\
\text { measured at shear rate }\end{array}$ & $\begin{array}{c}\text { Power-law fit curve for } \\
\text { viscosity versus shear rate }\end{array}$ & $R^{2}$ \\
\hline A & 23.5 & $42 \pm 1$ & 4.29 at $1870 \mathrm{~s}^{-1}$ & $\mu=53.485(\dot{\gamma})^{-0.344}$ & 0.994 \\
B & 27 & $34 \pm 1$ & 3.32 at $2381 \mathrm{~s}^{-1}$ & $\mu=11.099(\dot{\gamma})^{-0.172}$ & 0.861 \\
$\mathrm{C}$ & 27.1 & $37.5 \pm 1$ & 3.57 at $2352 \mathrm{~s}^{-1}$ & $\mu=9.193(\dot{\gamma})^{-0.13}$ & 0.9672 \\
$\mathrm{D}$ & 26 & $44 \pm 1$ & 4.12 at $2472 \mathrm{~s}^{-1}$ & $\mu=8.783(\dot{\gamma})^{-0.106}$ & 0.9442 \\
$\mathrm{E}$ & 26 & $44 \pm 1$ & 3.91 at $2536 \mathrm{~s}^{-1}$ & $\mu=15.107(\dot{\gamma})^{-0.185}$ & 0.9713 \\
$\mathrm{~F}$ & 25.6 & $44 \pm 1$ & 4.08 at $2479 \mathrm{~s}^{-1}$ & $\mu=9.462(\dot{\gamma})^{-0.116}$ & 0.9476 \\
\hline \hline
\end{tabular}

processes are short, the effect of any rheological behavior (viscous or non-Newtonian) is always negligibly small compared to the pressure impulse, and only the inertial effects play a role, while the initial flow field is potential, with the potential being determined by the impulse [24]. Then, under the conditions of the Lagrange theorem, which are fully applicable in the present case, the flow field will stay potential during its subsequent evolution [25]. Therefore, the splash of blood resulting from a bullet impact can effectively be considered in the framework of the inviscid potential flow approximation, as in the classical Wagner problem [24-29].

In the experiments in Sec. II a bullet impacted a blood-impregnated sponge with open porosity and very low hydraulic resistance. The situation therefore effectively resembles the situation of a bullet impacting a pool of blood, which then splashes. It is assumed that there is no significant difference whether blood is being emitted from a free surface or from a highly porous sponge with open porosity during the stage of interest when pressure is high and flow processes are short. It is also assumed that the mechanical behavior of a real skin (a porous organ containing blood vessels including capillaries) hardly matters and thus blood splashing from a free surface seems to be a plausible model of real gunshot back spatters.

Denote the flow potential by $\varphi$ and consider the axisymmetric Laplace equation, which it should satisfy in blood back-spatter flow generated by a bullet impact

$$
\nabla^{2} \varphi=\frac{1}{r} \frac{\partial}{\partial r}\left(r \frac{\partial \varphi}{\partial r}\right)+\frac{\partial^{2} \varphi}{\partial z^{2}}=0
$$

with $r$ and $z$ being the radial and axial coordinates, respectively. Note that the $z$ axis corresponds to the direction of bullet impact and is directed from the free surface into the liquid bulk.

The front part of the bullet used in the experimental part of the present work is a truncated cone, which is a transitional shape between a cone and a blunt body. In the present work, the theoretical approach is developed and tested for a purely conical bullet shape (see Fig. 3) as a first approximation. A theory for a blunt bullet impact is beyond the scope of the present work. It should be emphasized that in both cases (available in reality, separately, or as transitional shapes) back spatter of blood will be traced to the Rayleigh-Taylor instability, as is shown below.

In the present case a bullet is considered as a slender body of revolution and effectively acts as a system of sources of strength $q$ distributed along its main axis. In such cases the resulting potential can be found as [25]

$$
\varphi(r, z)=-\frac{1}{4 \pi} \int \frac{q(\xi)}{\sqrt{(\xi-z)^{2}+r^{2}}} d \xi,
$$

where the integration is conducted over the bullet axis, with $\xi$ being the axial dummy variable and $q$ a function of the velocity and shape of the bullet.

When the bullet tip has reached depth $h(t)$ below the free surface, with $t$ being time, the condition that the surface is free is accommodated by the mirror reflection of the submerged conical part of 
(a)

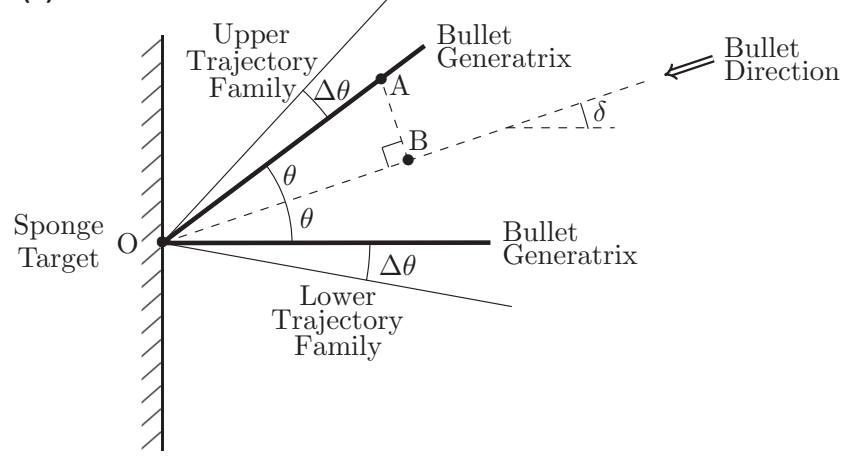

(b)

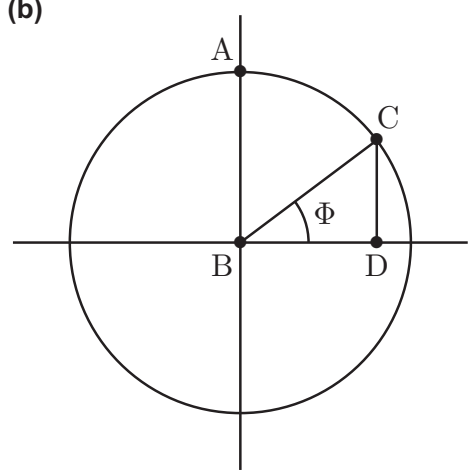

FIG. 3. (a) Origin of trajectory families of blood drops in back spatter. Only two trajectory families corresponding to $\Phi=\pi / 2$ (the uppermost trajectory family) and $\Phi=3 \pi / 2$ (the lowermost trajectory family) are shown. (b) Normal cross section of the conical tip with the azimuthal angle $\Phi$.

the bullet. This allows one to introduce the integration limits as follows:

$$
\varphi(r, z)=-\frac{1}{4 \pi} \int_{-h}^{h} \frac{q(\xi)}{\sqrt{(\xi-z)^{2}+r^{2}}} d \xi .
$$

It should be emphasized that the free surface is assumed to be located at $z=0$, approximately at the same place where it was at the moment of the bullet impact, which is a good approximation for sufficiently slender bullets.

The strength of the sources distributed over the submerged and mirror-reflected axis of the bullet is established using the fact that the bullet surface is impenetrable for blood and thus

$$
\left.\frac{\partial \varphi}{\partial r}\right|_{\text {bullet surface }}=\frac{1}{2 \pi} \frac{q(\xi)}{\left.r\right|_{\text {bullet surface }}} .
$$

Since at the bullet surface the normal component of blood motion is equal to the normal component of the bullet velocity, one can find from Eq. (4) that

$$
\frac{1}{2 \pi} \frac{q(\xi)}{\left.r\right|_{\text {bullet surface }}}= \begin{cases}\theta \dot{h} & \text { for } 0 \leqslant \xi \leqslant h \\ -\theta \dot{h} & \text { for }-h \leqslant \xi \leqslant 0,\end{cases}
$$

where the dot over $h$ denotes time differentiation and $\theta$ is the semiangle of the bullet tip. Accordingly,

$$
q(\xi)= \begin{cases}\left.2 \pi r\right|_{\text {bullet below surface }} \times \theta \dot{h} & \text { for } 0 \leqslant \xi \leqslant h \\ \left.2 \pi r\right|_{\text {bullet above surface }} \times(-\theta \dot{h}) & \text { for }-h \leqslant \xi \leqslant 0 .\end{cases}
$$

Note that the conical geometry of the bullet tip yields

$$
\begin{array}{ll}
\left.r\right|_{\text {bullet below surface }}=(h-\xi) \tan \theta \approx(h-\xi) \theta & \text { for } 0 \leqslant \xi \leqslant h, \\
\left.r\right|_{\text {bullet above surface }}=(h+\xi) \tan \theta \approx(h+\xi) \theta & \text { for }-h \leqslant \xi \leqslant 0 .
\end{array}
$$

Note also that Eq. (7) becomes less accurate for bullet tips significantly different from a thin cone, albeit still qualitatively true. Then Eqs. (6) and (7) yield

$$
q(\xi)= \begin{cases}2 \pi \theta^{2}(h-\xi) \times \dot{h} & \text { for } 0 \leqslant \xi \leqslant h \\ -2 \pi \theta^{2}(h+\xi) \times \dot{h} & \text { for }-h \leqslant \xi \leqslant 0 .\end{cases}
$$

After the distribution of the sources (and also sinks corresponding to the mirror image of the submerged bullet tip) are found, Eqs. (3) and (8) result in the following expression for the flow 
potential:

$$
\varphi(r, z)=-\frac{1}{2} \theta^{2} \dot{h} \int_{0}^{h} \frac{h-\xi}{\sqrt{(\xi-z)^{2}+r^{2}}} d \xi+\frac{1}{2} \theta^{2} \dot{h} \int_{-h}^{0} \frac{h+\xi}{\sqrt{(\xi-z)^{2}+r^{2}}} d \xi .
$$

Blood velocity at the free surface (where it is splashed against the direction of bullet motion) is found as

$$
\left.v_{z}\right|_{z=0}=\left.\frac{\partial \varphi}{\partial z}\right|_{z=0}=-\theta^{2} \dot{h}\left[\frac{h}{r}+\ln \frac{r}{h+\sqrt{h^{2}+r^{2}}}\right] .
$$

For example, at the free surface in contact with the penetrating bullet, i.e., at $r=\theta h$, at the moment of impact when $\dot{h}=V_{0}$, with $V_{0}$ being the bullet impact velocity, Eq. (10) yields

$$
\left.\frac{\partial \varphi}{\partial z}\right|_{z=0, r=\theta h}=-\theta^{2} \dot{h}\left[\frac{1}{\theta}+\ln \frac{\theta}{1+\sqrt{1+\theta^{2}}}\right] \approx-\theta \dot{h}=-\theta V_{0} .
$$

This back-spatter velocity is negative since it is directed against the positive bullet velocity $V_{0}$ and, moreover, it is much lower than the latter, since for slender bullets $\theta \ll 1$. If one takes for the estimate $\theta=0.24$ (which corresponds to the semiangle of $14^{\circ}$ ) and a bullet velocity of $340 \mathrm{~m} / \mathrm{s}$, the initial back-spatter velocity is $\sim 80 \mathrm{~m} / \mathrm{s}$.

Evaluating the integrals in Eq. (9) and using the first of Eqs. (7) allows one to find the potential distribution at the surface of the submerged bullet tip

$$
\left.\varphi\right|_{\text {bullet surface }}=-\frac{1}{2} \theta^{2} \dot{h}\left[-2(h-z) \ln \theta+2 z+(h-z) \ln 4+h \ln \left(\frac{z^{2}}{h^{2}-z^{2}}\right)+z \ln \left(\frac{h-z}{h+z}\right)\right] .
$$

The nonsteady Bernoulli equation in the slender-body approximation yields the pressure $p$ at the bullet surface as

$$
\left.p\right|_{\text {bullet surface }}=-\rho \frac{\left.\partial \varphi\right|_{\text {bullet surface }}}{\partial t}
$$

where $\rho$ is the blood density. The drag force $F$ experienced by the penetrating bullet in the slenderbody approximation is found using the integral over the axially symmetric wetted surface of a slender conical bullet

$$
F=-\left.2 \pi \theta^{2} \int_{0}^{h} p\right|_{\text {bullet surface }}(h-z) d z
$$

Then Eqs. (12)-(14) yield

$$
F=\lambda \pi \theta^{4} \rho(\dot{h})^{2} h^{2}+\lambda \frac{\pi}{3} \theta^{4} \rho(\ddot{h}) h^{3},
$$

where the dimensionless factor

$$
\lambda=\ln 2 \theta+1 .
$$

It should be emphasized that for a slender bullet $\lambda<0$ and thus the force $F<0$, i.e., acting against the direction of bullet motion. Note also that the first term on the right-hand side in Eq. (15) expresses the shape drag, whereas the second one expresses the added-mass drag.

The second law of Newton for the bullet motion reads

$$
m \ddot{h}=F,
$$


where $m$ is the bullet mass. Substituting the expression for the drag force from Eq. (15) into Eq. (17), the equation describing bullet motion when it penetrates the blood is found

$$
\frac{d^{2} h}{d t^{2}}=\frac{\lambda \pi \theta^{4} \rho(d h / d t)^{2} h^{2}}{m-\lambda \pi \theta^{4} \rho h^{3} / 3}
$$

which should be solved subjected to the initial conditions

$$
t=0: \quad h=0, \quad \frac{d h}{d t}=V_{0}
$$

Using Eq. (10), the acceleration of the free liquid surface $A$ is found as

$$
A=\frac{\left.\partial v_{z}\right|_{z=0}}{\partial t}=-\theta^{2} \frac{d^{2} h}{d t^{2}}\left[\frac{h}{r}+\ln \frac{r}{h+\sqrt{h^{2}+r^{2}}}\right]-\theta^{2}\left(\frac{d h}{d t}\right)^{2}\left[\frac{1}{r}+\frac{1}{\sqrt{h^{2}+r^{2}}}\right] .
$$

The estimates based on Eqs. (18)-(20) show that at $d h / d t \sim V_{0}=340 \mathrm{~m} / \mathrm{s}$, the bullet deceleration $d^{2} h / d t^{2} \sim-121 \mathrm{~m} / \mathrm{s}^{2}$. Accordingly, for $h \sim r \sim 1 \mathrm{~cm}$, the second term on the right-hand side in Eq. (20) is much larger than the first one and thus the acceleration of the liquid surface that results in back spatter is

$$
A \approx-\theta^{2} V_{0}^{2}\left[\frac{1}{r}+\frac{1}{\sqrt{h^{2}+r^{2}}}\right] \approx-\frac{\theta^{2} V_{0}^{2}}{r} .
$$

It should be emphasized that blood moves with the acceleration $A$ toward air. Since the blood density is higher than the air density, such flow is prone to the Rayleigh-Taylor instability [30]. Accordingly, the fastest growing wavelength of such instability that determines the drop size $l_{*}$ can be found as

$$
l_{*}=\frac{2 \pi}{\sqrt{\rho|A(r)| / 3 \sigma}} w
$$

(with $w$ being a dimensionless factor), whereas the drop velocity, according to Eq. (10), is given by

$$
\left.v_{z}\right|_{z=0}=-\theta^{2} V_{0} \frac{h}{r}
$$

Note that in Eqs. (21)-(23) $r \geqslant \theta h$. Note also that the fastest growing wavelength of the RayleighTaylor instability determines only the order of magnitude of the drop size, while the factor $w$, which is of the order of 1, is needed in Eq. (22) for an exact match. Its choice is discussed in Sec. IV.

Equation (23) shows that the most important information pertaining to the bullet is the semiangle of its tip $\theta$ and the bullet impact velocity $V_{0}$. Sensitivity of the results to the uncertainty in these values is not presently addressed and is considered as a task for future work.

Denote the blood drops originating from the zone $b_{i} \leqslant r / h \leqslant b_{i+1}$ (with $b_{i}$ being dimensionless numbers increasing with $i$ ) as a bin $i$. The mass $M_{i}$ of the blood drops in the back spatter originating from this bin during time $\tau=c h / V_{0}$ is found as

$$
M_{i}=\tau \int_{b_{i}}^{b_{i+1}}\left|v_{z}\right|_{z=0} \mid 2 \pi r d r=2 \pi \rho(h \theta)^{2}\left(b_{i+1}-b_{i}\right) c
$$

(with $c$ being a dimensionless factor), whereas the number of drops $n_{i}$ in such a bin is approximately equal to

$$
n_{i}=\frac{M_{i}}{\rho \pi l_{*_{i}}^{3} / 6}
$$

where the characteristic drop size $l_{*_{i}}$ is assumed to be the diameter. Note that the characteristic time of the back spatter could be taken as the characteristic time of the Rayleigh-Taylor instability, which would yield in the present case $\tau \sim(\sigma / \rho)^{1 / 4} h^{3 / 4} / V_{0}^{3 / 2}$. This estimate is, however, quite uncertain, 
since multiple-drop formation might take several characteristic times and be different for different radial coordinates $r$. Therefore, a uniformly valid time scale $\tau=c h / V_{0}$ is used, which introduced the factor $c$ in Eq. (24). Its choice is discussed in Sec. IV.

\section{B. Trajectories in the blood drop cloud}

One can assume that drops of different size groups and initial velocities form subfamilies (bins, as termed in the previous section), each following different trajectories. This means that the original cloud of blood drops of different sizes resulting from the Rayleigh-Taylor instability consists not of a continuous spectrum of drop sizes, but of an approximate discrete one, which is a reasonable approximation when many different sizes are involved. In other words, a discrete distribution replaces the so-called diffusion approximation for the drop-size distribution. Then each group of drops (each bin) interacts with the other groups through air and develops its own trajectory according to the laws of mechanics. Consider one of these bins and pose the problem that allows one to describe its trajectory in back spatter starting from the initial wound. Assume that drops of this size group form a continuum that can be considered as a pseudogas moving through air (it should be emphasized that this pseudogas possesses an effective density but not a viscosity). Then air and the pseudogas form a system of two interpenetrating continua, which is known to be an effective approach in the fluid mechanics of two-phase flows [31]. Denote a parcel of drops originating from a bin by $i$ and moving with an entrained air as a blob $i$. Introduce the instantaneous averaged velocity of the blob $i$ by $u_{i}$ and the instantaneous averaged velocity of air accompanying this blob due to the entrainment in motion by all its drops by $U_{i}$. The interaction of an individual drop with the surrounding air corresponds to the air drag force $F_{D, i}$, which can be introduced using the drag coefficient $C_{D, i}[31]$,

$$
C_{D, i}=\frac{F_{D, i}}{(1 / 2) \rho_{a}\left(u_{i}-U_{i}\right)\left|u_{i}-U_{i}\right| \pi l_{*_{i}}^{2}},
$$

where $\rho_{a}$ is the air density. The drag coefficient is a function of the Reynolds number for an individual drop $\operatorname{Re}_{i}=u_{i} 2 l_{*} / v_{a}$, with $v_{a}$ being the kinematic viscosity of air. The following correlation for the drag coefficient is used in the present case and applied to individual drops in the blob $i[3,32]$ :

$$
C_{D, i}=0.28+\frac{6}{\mathrm{Re}_{i}^{0.5}}+\frac{21}{\mathrm{Re}_{i}} \text { for } 0.1 \leqslant \mathrm{Re}_{i} \leqslant 4000 .
$$

This formula is used in BPA research and other fluid mechanical applications [3,32] and its accuracy and the range of validity is comparable to those of the standard drag curve given by the Schiller-Neumann formula. Note also that in the simulations discussed in Sec. IV the values of $\operatorname{Re}_{i}$ were always in the range of validity of Eq. (27), from 0.1 to 4000 .

Then the momentum balance of air in the air-drop blob projected onto the tangent of the trajectory is given by

$$
\frac{d}{d t}\left(\rho_{a} V U_{i}\right)=\sum_{i} n_{i} C_{D, i} \frac{1}{2} \rho_{a}\left(u_{i}-U_{i}\right)\left|u_{i}-U_{i}\right| \pi l_{*_{i}}^{2} .
$$

Here $V$ is the volume of air in the air-drop blob. It should be emphasized that the effect of gravity on air motion is immaterial compared to the drop motion discussed below, i.e., the gravity effect on the motion of an air-drop blob is determined by its effect on the drop motion, while air is entrained via the interfacial drag force as in Eq. (28) and the viscous suction of air into the blob discussed below.

The momentum balance of drops of a chosen size group in the air-drop blob is

$$
M_{i} \frac{d u_{i} \boldsymbol{\tau}}{d t}=-F_{D, i} \boldsymbol{\tau}-M_{i} g \mathbf{k},
$$


where $\boldsymbol{\tau}$ is the unit vector tangent to the trajectory, $g$ is gravity acceleration, and $\mathbf{k}$ is the unit vector of the vertical direction. Projecting Eq. (19) onto the tangent $\boldsymbol{\tau}$ to the trajectory yields

$$
M_{i} \frac{d u_{i}}{d t}=-n_{i} C_{D, i} \frac{1}{2} \rho_{a}\left(u_{i}-U_{i}\right)\left|u_{i}-U_{i}\right| \pi l_{*_{i}}^{2}-M_{i} g \frac{d H}{u_{i} d t},
$$

where $H$ is the current elevation of the blob relative to the ground. On the other hand, the momentum balance of drops of a chosen size group (bin) in the air-drop blob [Eq. (29)] projected onto the normal to the trajectory yields

$$
\frac{d^{2} H}{d t^{2}}=-g \frac{\left[u_{i}^{2}-(d H / d t)^{2}\right]}{u_{i}^{2}}+\frac{1}{u_{i}} \frac{d H}{d t} \frac{d u_{i}}{d t} .
$$

A moving air-drop blob acts on the surrounding air as an axisymmetric air jet occupying the same space. The outside air experiences suction toward the jet core sustained by the viscous forces. The mass of air passing through the jet cross section in unit time $G$ increases due to the suction proportional to the location along the trajectory characterized by the arc length $\xi$ [33],

$$
G=2 \pi \rho_{a} \int_{0}^{\infty} U y d y=8 \pi \mu_{a} \xi
$$

where the radial coordinate reckoned from the trajectory is denoted by $y$ and $\mu_{a}$ is air viscosity. The volumetric flux of air through the jet cross section $\dot{V}$ is thus equal to

$$
\dot{V}=\frac{G}{\rho_{a}}=8 \pi v_{a} \xi
$$

with $v_{a}=\mu_{a} / \rho_{a}$ being the kinematic viscosity of air.

An air-drop blob has a characteristic size of $V^{1 / 3}$ and it takes time $V^{1 / 3} / u_{i}$ to pass the cross section. Therefore, $V=\dot{V}\left(V^{1 / 3} / u_{i}\right)$ and thus the blob volume increases in time as

$$
V(t)=\left[8 \pi v_{a} \frac{\xi(t)}{u_{i}(t)}\right]^{3 / 2} .
$$

In turbulent jetlike motion the kinematic viscosity $v_{a}$ is in fact the eddy viscosity. In the framework of the semiempirical Prandtl mixing length theory it is possible to show that $v_{a}=\kappa_{1} u_{i 0, \max } V_{\text {blood }}^{1 / 3}$, where $u_{i 0 \text {, max }}$ and $V_{\text {blood }}$ are the maximal initial velocity of a drop bin and volume of blood in the back spatter $\left(V_{\text {blood }}=\sum_{i} M_{i} / \rho\right)$, respectively, and the semiempirical constant $\kappa_{1}=0.16-0.19$ [33]. Accordingly, Eq. (34) for the air-drop volume can be transformed to

$$
\frac{V(t)}{V_{\text {blood }}}=\kappa\left[\frac{\xi(t)}{V_{\text {blood }}^{1 / 3}} \frac{u_{i 0, \max }}{u_{i}(t)}\right]^{3 / 2}, \quad \kappa=0.255-0.33 .
$$

The current arc length corresponding to the blob location along the trajectory is found as

$$
\frac{d \xi}{d t}=u_{i}
$$

and the current horizontal coordinate of the blob is governed by the equation

$$
\frac{d X}{d t}=\sqrt{\left(\frac{d \xi}{d t}\right)^{2}-\left(\frac{d H}{d t}\right)^{2}} .
$$

The six equations (28), (30), (31), and (35)-(37) govern the motion of each blob and allow one to predict all six unknown functions $U_{i}(t), u_{i}(t), V(t), \xi(t), H(t)$, and $X(t)$ and thus to determine the trajectory as $H=H(X)$ from its origin at a bullet wound to a solid surface onto which the blob impacts as a result of back spatter. It should be emphasized that blobs with drops of different sizes will 
follow different trajectories and will be deposited at different places, thus forming a blood-spatter pattern and the above-mentioned equations determine their mutual interaction (through air) in flight.

Having in mind a numerical solution of the system of governing equations, the equations are rendered dimensionless using the following scales: The initial drop velocity (determined by the Rayleigh-Taylor instability discussed in Sec. III A) $u_{i 0, \max }=\theta^{2} V_{0} / b_{1}$ (the maximal initial velocity corresponds to bin $i=1$ ) is used as a scale for air and drop velocities $U_{i}$ and $u_{i}$; the initial volume of the drop blob (also determined by the Rayleigh-Taylor instability discussed in Sec. III A) $V_{\text {blood }}^{1 / 3}$ is used as a scale for the arc length $\xi$, the horizontal and vertical locations of the blob $X$ and $H$, respectively, and for the drop size $l_{*_{i}}$ (also determined by the Rayleigh-Taylor instability discussed in Sec. III A); $V_{\text {blood }}$ is used as a scale for the air volume in the blob $V ; V_{\text {blood }}^{1 / 3} / u_{i 0, \text { max }}$ is used as a scale for time $t$. Dropping overbars over most of the dimensionless parameters for brevity, Eqs. (28), (30), (31), and (35)-(37) are transformed to the dimensionless form

$$
\begin{gathered}
\frac{d U_{i} V}{d t}=\left(\sum_{i=1}^{N} n_{i}\right) \frac{1}{2} C_{D, i}\left(u_{1}-U_{i}\right)\left|u_{1}-U_{i}\right| \pi \bar{l}_{*_{i}}^{2}, \\
\frac{d u_{i}}{d t}=-\frac{1}{2} n_{i} C_{D, i} \frac{\rho_{a}}{\rho}\left(u_{i}-U_{i}\right)\left|u_{i}-U_{i}\right| \pi \bar{l}_{*_{i}}^{2}-\frac{1}{\operatorname{Fr}^{2}} \frac{f}{u_{i}}, \\
\frac{d H}{d t}=f, \\
\frac{d f}{d t}=-\frac{1}{\operatorname{Fr}^{2}} \frac{\left(u_{i}^{2}-f^{2}\right)}{u_{i}^{2}}+\frac{f d u_{i} / d t}{u_{i}}, \\
V(t)=\kappa\left[\frac{\xi(t)}{u_{i}(t)}\right]^{3 / 2}, \quad \kappa=0.255-0.33, \\
\frac{d X}{d t}=\sqrt{\left(\frac{d \xi}{d t}\right)^{2}-\left(\frac{d H}{d t}\right)^{2}} .
\end{gathered}
$$

It should be emphasized that Eq. (38) approximately expresses the fact that air entrainment is dominated by the fastest moving drops "leading" the cloud, as was previously demonstrated regarding the two-phase flows characteristic of sprinkler jets and diesel sprays [21,22]. A similar phenomenon is commonly implied regarding the formation flight (or $V$ formation) of a geese flock [34]. Equation (38) is integrated directly for the product $U_{i} V$. Also, $f$ in Eqs. (40) and (41) is an auxiliary variable used to split the second-order equation into two first-order ordinary differential equations.

The governing equations (38)-(44) and the expression (27) incorporate the following dimensionless groups, the Reynolds and the Froude numbers, Re and Fr, respectively:

$$
\operatorname{Re}_{i}=\operatorname{Re}_{0} u_{i} \bar{l}_{*_{i}} \quad \text { for } \operatorname{Re}_{0}=\frac{u_{i 0, \max } 2 V_{\text {blood }}^{1 / 3}}{v_{a}}, \quad \mathrm{Fr}^{-2}=\frac{g V_{\text {blood }}^{1 / 3}}{u_{i 0, \max }^{2}}
$$

where $\bar{l}_{*_{i}}=l_{*} / V_{\text {blood }}^{1 / 3}$. Solutions of Eqs. (38)-(44) are subjected to the initial conditions

$$
t=0: \quad U_{i}=0, \quad u_{i}=\frac{u_{i 0}}{u_{i 0, \max }}, \quad \xi=0, \quad X=0, \quad H=\overline{H_{0}}, \quad f=\frac{u_{i 0}}{u_{i 0, \max }} \sin \psi, \quad V=0,
$$

where $\overline{H_{0}}=H_{0} / V_{\text {blood }}^{1 / 3}$, with $H_{0}$ being the initial elevation and $\psi$ the initial inclination angle from the horizontal. More details about $\psi$ are given in the following section. The governing equations (38)-(44) with the initial conditions (46) are solved numerically using the Kutta-Merson method 
with an automatically chosen and variable time step depending on the requested accuracy of four digits after the decimal point.

\section{Sideways dispersion of back-spatter blood drops}

To predict the sideways dispersion of a blood spatter, consider a circular cross section of the conical part of a bullet by a surface normal to its main axis. Denote by $\Phi$ the angular positions over the circle, with $\Phi=0$ corresponding to the rightmost point on the right-hand side, $\Phi=\pi / 2$, to the uppermost point on the top, etc. Blood drops are spattered in planes incorporating the cone generatrices, while each such plane corresponds to a given value of $\Phi$. The splash direction at the moment of impact can differ from the bullet generatrix by an angle $\Delta \theta$ due to the flowerlike opening of hollow-point bullets, bullet mushrooming, the effect of the sponge (and skin, in reality), etc. (see Fig. 3).

Assume first that blood is splashed exactly over the generatrix of the conical part of the bullet, i.e., $\Delta \theta=0$. Then, by straightforward geometrical considerations (see Fig. 3) it is possible to show that the trajectory family corresponding to a certain value of $\Phi$ has the initial angle of inclination to the horizon $\psi$ given by the formula

$$
\psi=\psi_{0}+\Delta \psi
$$

where the following notation for $\psi_{0}$ and $\Delta \psi$ is adopted:

$$
\sin \psi_{0}=\sin \theta \sin \Phi, \quad \sin \Delta \psi=\frac{\sin \delta}{\sqrt{1+\tan ^{2} \theta \cos ^{2} \Phi}} .
$$

Equations (47) and (48) finalize the initial conditions (46). Then trajectories of drops corresponding to all bins $i$ (having the same initial angle of inclination $\psi$ for any direction $\Phi$ of interest) can be found by numerical integration of the system (38)-(44) subjected to the initial conditions (46). Thus, for each drop its horizontal landing distance $X_{\Phi}$ from the origin can be predicted. The corresponding $X$ and $Y$ coordinates on the floor [the longitudinal coordinate $X$ belongs to the central plane ( $\Phi=\pi / 2$ or $\Phi=3 \pi / 2$ ), while $Y$ is the lateral coordinate reckoned from this plane (see Fig. 2)] are found as

$$
\begin{gathered}
X=X_{\Phi} \frac{\cos \delta \cos \theta}{\sqrt{\cos ^{2} \delta \cos ^{2} \theta+\sin ^{2} \theta \cos ^{2} \Phi}}, \\
Y=X \frac{\tan \theta \cos \Phi}{\cos \delta} .
\end{gathered}
$$

It should be emphasized that if $\Delta \theta \neq 0$, then $\theta$ in Eqs. (49) and (50) should be replaced by $\pm(\theta+\Delta \theta)$ for the upper and lower trajectory families, respectively [see Fig. 3(a)].

\section{RESULTS AND DISCUSSION}

There are at least three main parameters of interest arising from the experimental sets of data that characterize back spatter resulting from a gunshot. These data are the total number of stains, the average areas of the individual stains, and the total stain area, which are all functions of the drop landing position. Also, the drop impact angle and the corresponding Weber number are the important parameters to be determined. In addition, the cumulative percentages of different parameters are of significant interest, as discussed below. Each experimental trial described in Sec. II had its back-spatter stain pattern virtually dissected into strips normal to the projection of the bullet trajectory on the floor at certain locations from the target (see Fig. 4). This means that along this trajectory the floor was covered with paper incorporating all of these strips that collected blood drops landing there. The data collected on each strip for a certain parameter were lumped as a data point corresponding to a certain distance from the target. Counting the number of stains and measuring their respective area on each strip produced an average value and standard deviation of the data point resulting from such a strip. The strips were discretized such that the uncertainty of the measurements 


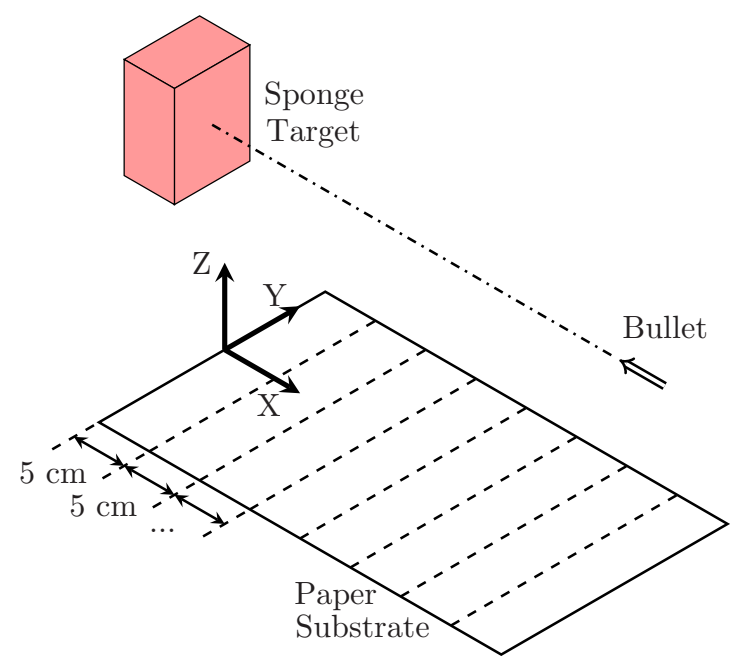

FIG. 4. Strips used for the processing of the experimental data. The strips were chosen to minimize uncertainty by being as small as possible, yet not too small to become meaningless. It was found that $5-\mathrm{cm}$ strips (in the $X$ direction) are the optimal size.

of parameters of interest is minimized and the resolution remains fine enough to create a wide range of data. In particular, both the experimental (and the corresponding theoretical) strips were $5 \mathrm{~cm}$ wide in the direction from the target to the shooter.

Additionally, the cumulative percentage plots based on the experimental data (not dissected into strips) are of interest for the BPA community:

$$
C=\frac{\sum_{j=1}^{K \leqslant N} P_{j}}{\sum_{j=1}^{N} P_{j}} 100 \%,
$$

where $C$ denotes the cumulative percentage, $P_{j}$ is the parameter of which the cumulative percentage is being calculated, $N$ is the total number of data values for this parameter, and $K$ is the current boundary parameter (less than or equal to $N$, which forces the last value for the cumulative percentage to always be $\leqslant 100 \%$ ). Note that the parameter chosen for $P_{j}$ corresponds to the $j$ th landing location on the ground, in order of $X$ distance to the source. An advantage of cumulative distributions is that they do not use strips and do not depend upon image scanner resolution since weighting is done by the parameter of interest. The cumulative distribution is essentially invariant to the size of the minimum image pixel and therefore beneficial for BPA regardless of the equipment used.

To mitigate the experimental data noise due to processing aspects such as imaging resolution, only the bloodstains with an area greater than $0.375 \mathrm{~mm}^{2}$ are considered. For consistency, this rule was followed for both the experimental data as well as the theoretical predictions. The experimental stain area resulting from a single-drop impact was determined by the pixel density found via a purpose-built in-house program. The theoretically predicted stain area resulting from a single-drop impact is found from the spread factor [35-37]

$$
\chi=0.61\left(\frac{\mathrm{We}_{\text {impact }}}{\mathrm{Oh}}\right)^{0.166},
$$

where $\chi=D_{\max } / l_{*_{i}}$, with $D_{\max }$ being the final stain diameter and the final (impact) Weber and Ohnesorge numbers being, respectively,

$$
\mathrm{We}_{\text {impact }}=\frac{\rho l_{*_{i}} u_{i, \text { impact }}^{2}}{\sigma}, \quad \mathrm{Oh}_{\text {impact }}=\frac{\sqrt{\mathrm{We}_{\text {impact }}}}{\mathrm{Re}_{i}} .
$$




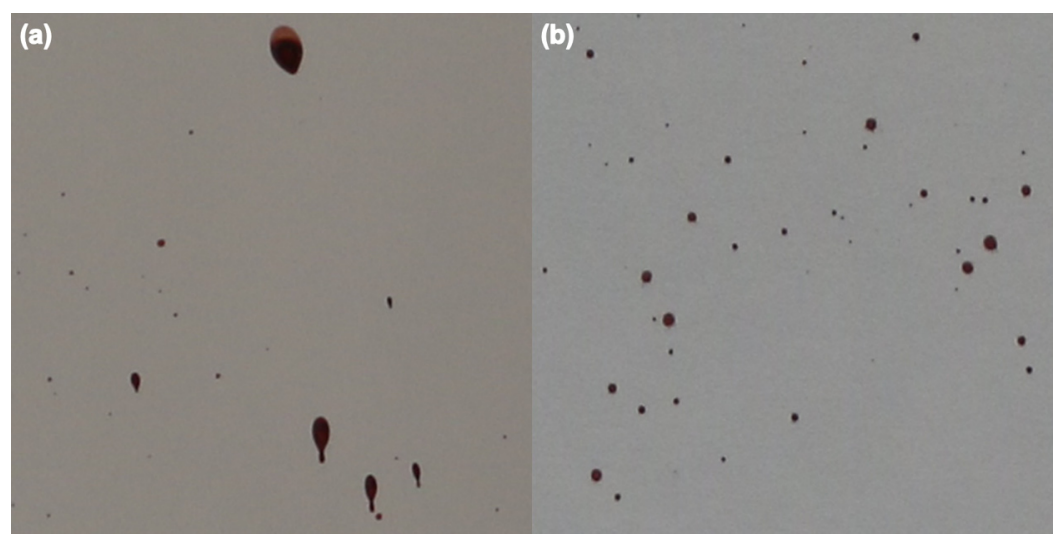

FIG. 5. (a) Experimental bloodstains in back spatter formed on a vertical paper sheet. (b) Horizontal paper sheet. Note that the elongated shape of some stains in (a) is mostly due to the angle of impact, rather than due to the drop sliding down the surface after impact.

Here $\rho$ and $\sigma$ are the density and surface tension of blood, respectively, and $\operatorname{Re}_{i}$ is the drop Reynolds number from the first part of Eq. (45). The impact velocity $u_{i \text {,impact }}$ is taken as the velocity magnitude, not the component that is normal to the impacted surface. The reason for this is that the normal component of velocity, $U_{H}$ for the horizontal deposition case, is largely due to the relatively large impact angles. These large angles mean that drops impact practically vertically and the stain should be almost circular. This is opposite of the vertical deposition cases where the normal component of velocity is $U_{V}$, which is small and the trajectory hits a vertical substrate tangentially creating elliptic stains. Therefore, the spread factor of Eq. (52) can be greatly underestimated if normal velocity components are used for the vertical deposition cases, since $U_{V}$ is much smaller than $U_{H}$. Experimental evidence supports the idea of using the impact velocity magnitude in Eqs. (52) and (53), since large elliptical stains are associated with the tangential velocity component, as Fig. 5 and [38] show. It should be emphasized that the relation between the ellipticity of bloodstains and their immediate deformation upon impact is known in forensic literature [4,6,7,11]. A comparison between computational fluid dynamics and high-speeed visualization of the deformation stages and final stain shape during oblique impact of blood drops is shown in Fig. 10 of [3]. As a matter of fact, there is a plausible relationship between the ellipticity of the stain and the angle of impact $[3,4,6,7,11]$. It should be emphasized that in the present experiments sufficient experimental data were collected only on the horizontal surface (the floor), therefore, only this case is discussed here.

Several governing parameters characteristic of the experiment, namely, bullet velocity and penetration depth into blood at the moment of backward splashing, as well as the semiangle of the bullet cone that changes during the impact of a hollow-point bullet (such as the one used here) are known only with an order-of-magnitude accuracy. Therefore, a single set of these parameters was chosen to uniformly match the horizontal data sets in the trial H-a0-D300, i.e., the data collected on the floor along the bullet trajectory when shooting was horizontal and parallel to the floor. The parameters found from this matching were the bullet impact velocity $V_{0} \approx 300 \mathrm{~m} / \mathrm{s}$, semiangle of the bullet cone $\theta \approx 0.16 \mathrm{rad}\left(\sim 9^{\circ}\right)$, the depth of the bullet penetration corresponding to the main blood splash $h \approx 1 \mathrm{~cm}$, the dimensionless factor in Eq. (22) $w=0.9$, and the dimensionless factor in Eq. (24) $c=0.001$. For both sets of comparisons this set of parameters was kept unchanged. Note also that the azimuthal angle over the cross section of the bullet $\Phi$ was set to increment at $\pi / 2$ around the entire cone.

The bins $b_{i} \leqslant r / h \leqslant b_{i+1}$ were chosen to be equal, since the majority of the drops inevitably result from a few bins sufficiently close to the line of the bullet intersection with the free surface encompassing the blood mass. Thus, the choice of these bins, rather than their different widths, was carefully explored in the preliminary simulations. 
(a)
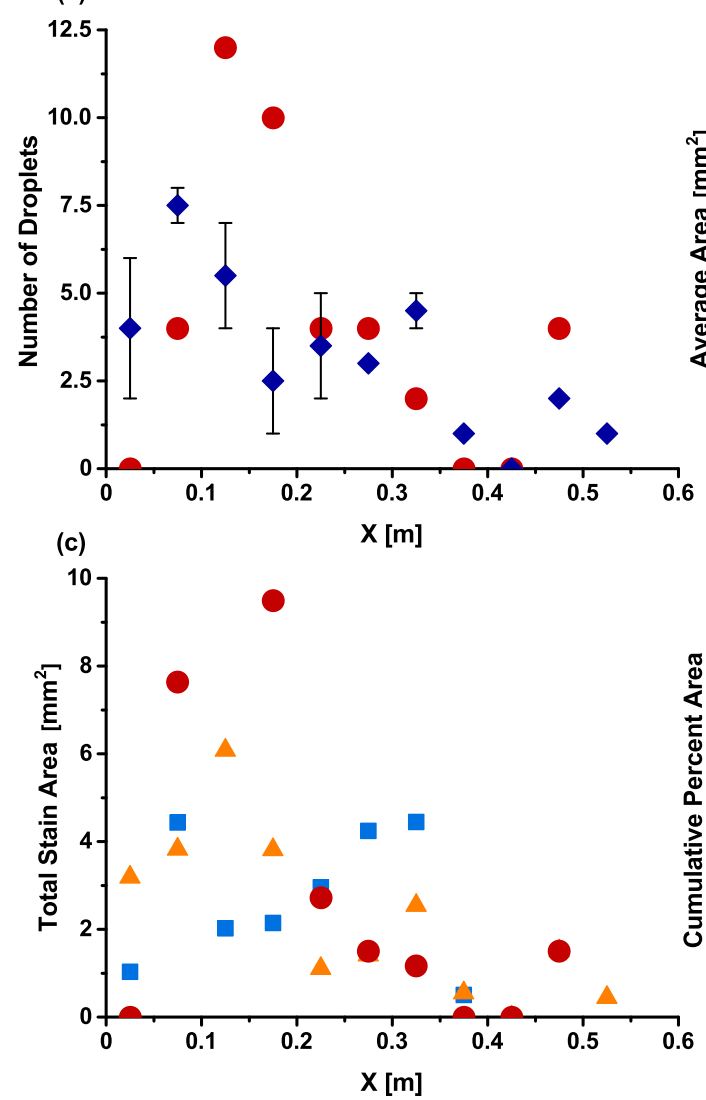

(b)
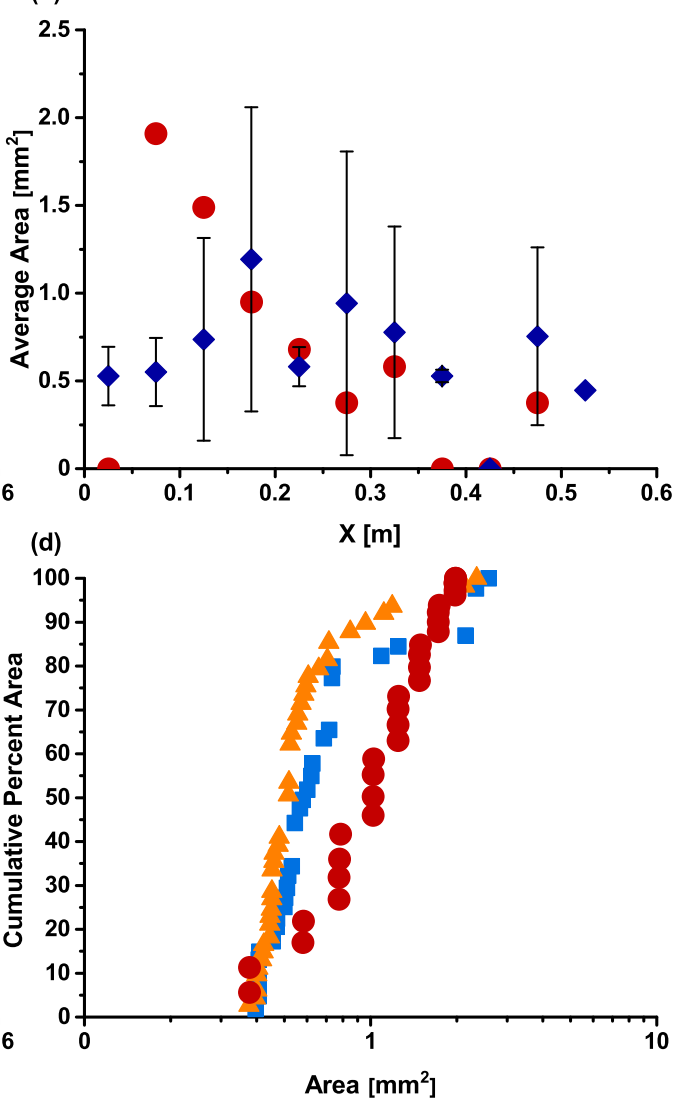

FIG. 6. Numerical predictions versus experimental data set $\mathrm{H}$-a0-D300 corresponding to the bullet impact angle $\delta=0^{\circ}$. The light blue squares represent experimental trial H-a0-D300-\#1, orange triangles represent experimental trial H-a0-D300-\#2, and red circles depict the numerical results. The dark blue diamonds represent the average of the two trials.

The splash direction can differ from the bullet generatrix due to the flowerlike opening of hollow-point bullets at the moment of impact, the effect of the sponge (and body, in reality), the effect of muzzle gases and the bullet wake [39], etc. Therefore, the deviation angle $\Delta \theta \approx 40^{\circ}$ was introduced (see Fig. 3). For example, this means that for the trajectory family corresponding to $\Phi=\pi / 2$ (the top generatrix of the bullet) the initial angle of inclination is $\psi=\delta+\theta+\Delta \theta$, whereas for the trajectory family corresponding to $\Phi=3 \pi / 2$ (the bottom generatrix of the bullet) the initial angle of inclination is $\psi=\delta-\theta-\Delta \theta$.

The comparison of the experimental data acquired on the floor in the experiments at two angles of bullet impact onto the target, namely, of the data sets H-a0-D300 and H-a60-D150, corresponding to $\delta=0^{\circ}$ and $60^{\circ}$, respectively, is shown in Figs. 5 and 6. Both figures have four panels, one for each of the parameters of interest as previously described, namely, panel (a) is the number of stains, panel (b) is the average stain area, panel (c) is the total stain area, all of which are functions of the final stain landing distance from the target, and panel (d) is the cumulative percentage for the stain area as a function of the individual drop stain area. The experimental data in panels (a) and (b) represent combinations of two trials under identical conditions, i.e., the dark blue diamonds were found by averaging the number of droplets or area on each strip and the error bars represent the corresponding standard deviation. 
(a)

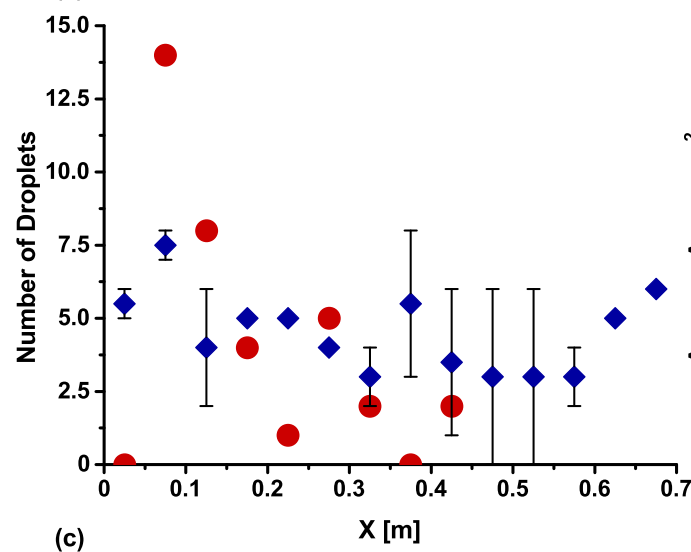

(c)

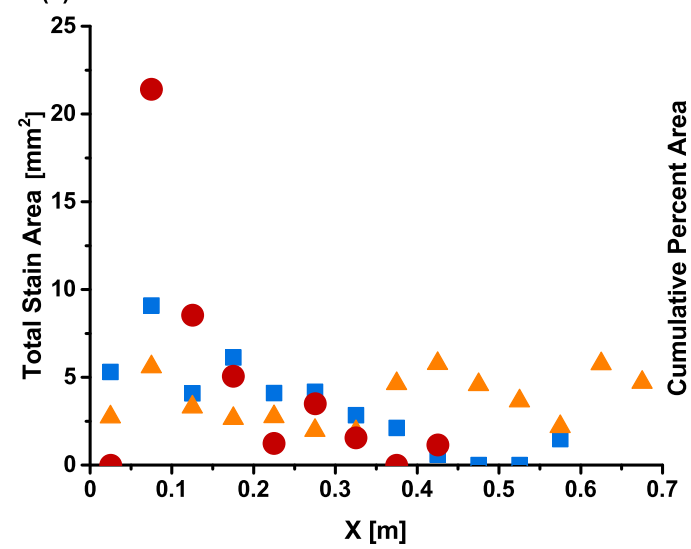

(b)

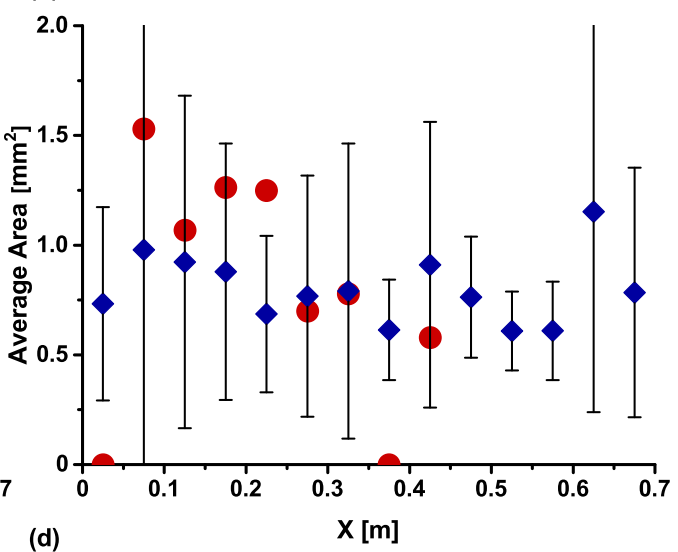

(d)

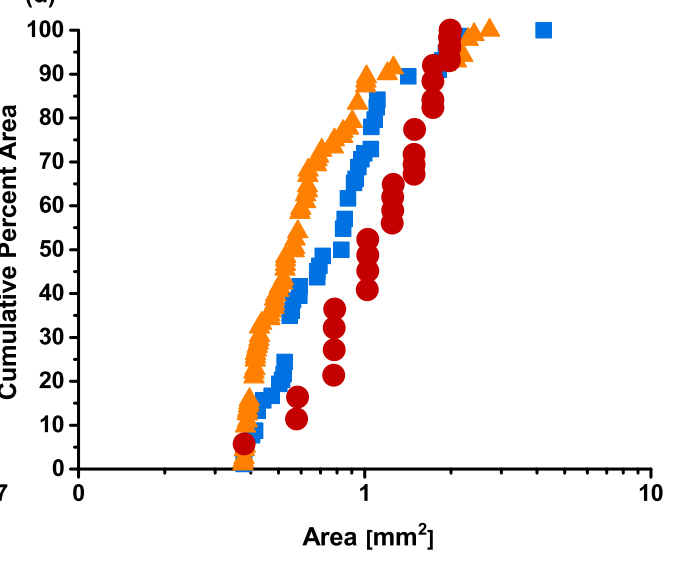

FIG. 7. Numerical predictions versus experimental data set $\mathrm{H}$-a60-D150 corresponding to the bullet impact angle $\delta=60^{\circ}$. The light blue squares represent experimental trial H-a60-D150-\#1, orange triangles represent experimental trial H-a60-D150-\#2, and red circles depict the numerical results. The dark blue diamonds represent the average of the two trials.

Figures 6 and 7 show a certain agreement (at least, at the order of magnitude) between the experimental and numerical data for all the parameters of interest. It should be emphasized that the comparison of the two experimental data sets corresponding to the same conditions and denoted by \#1 and \#2 reveals significant error bars for the data, practically incorporating the theoretical predictions [see Figs. 6(b) and 7(b)]. Note also that the theoretical predictions in Figs. 6(d) and 7(d) are serrated because of the discretization of the drop size $l_{*}$ [see Eqs. (21)-(24)] in the numerical simulations.

The cumulative percentage of Eq. (51) can be calculated for any property of interest, for example, for the number of stains and drop impact angle $\alpha$ (the notation recommended in Refs. [3,40]; see Fig. 1). The corresponding numerical results are shown together with the experimental data in Fig. 8, with the relatively low repeatability of the data from trial to trial under the same conditions clearly visible once again. Still, Figs. 8(a) and 8(b) show that the theory and the experiments are in generally good agreement, albeit the theory underpredicts the cumulative percentage of the number of stains at low values of $X$ close to the front surface of the target. This might stem from secondary effects such as the arrival of the elastic rarefaction wave from the rear surface of the target causing issuing of additional secondary blood ejecta, the effect not accounted for by the present theory.

The impact angle and drop impact Weber number $\mathrm{We}_{\text {impact }}$ are important for the BPA community since a set of approaches based on the analysis of these parameters has already been developed [3]. The theoretical predictions for the drop impact Weber number are shown in Fig. 9, where it can be 
(a)

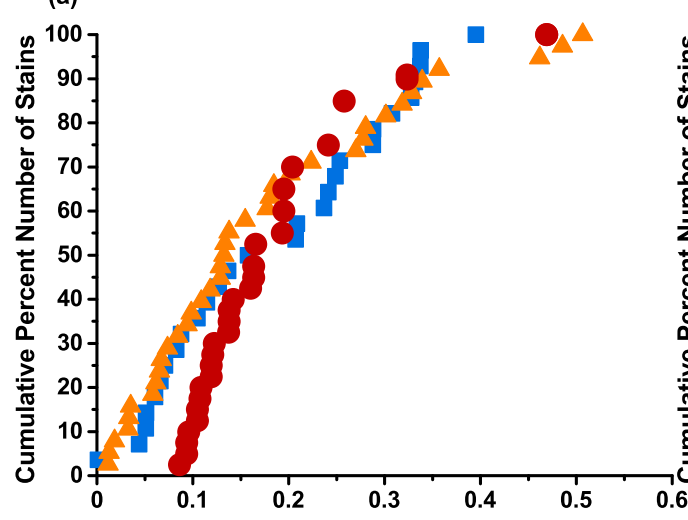

(c) $\quad X[\mathrm{~m}]$

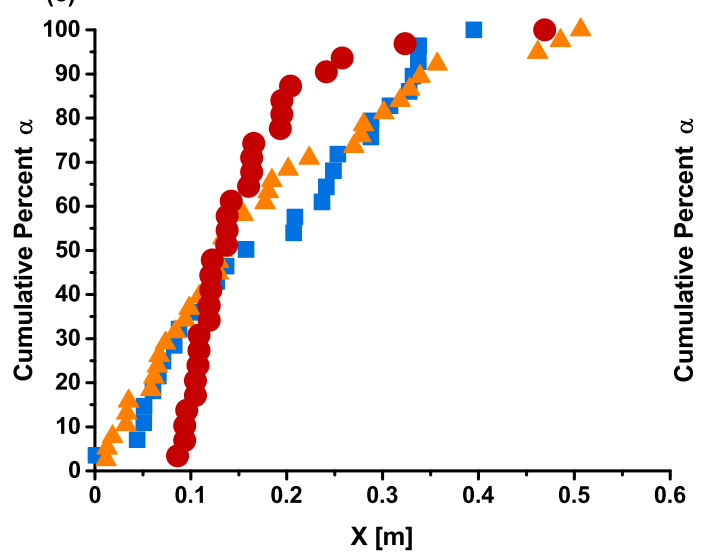

(b)
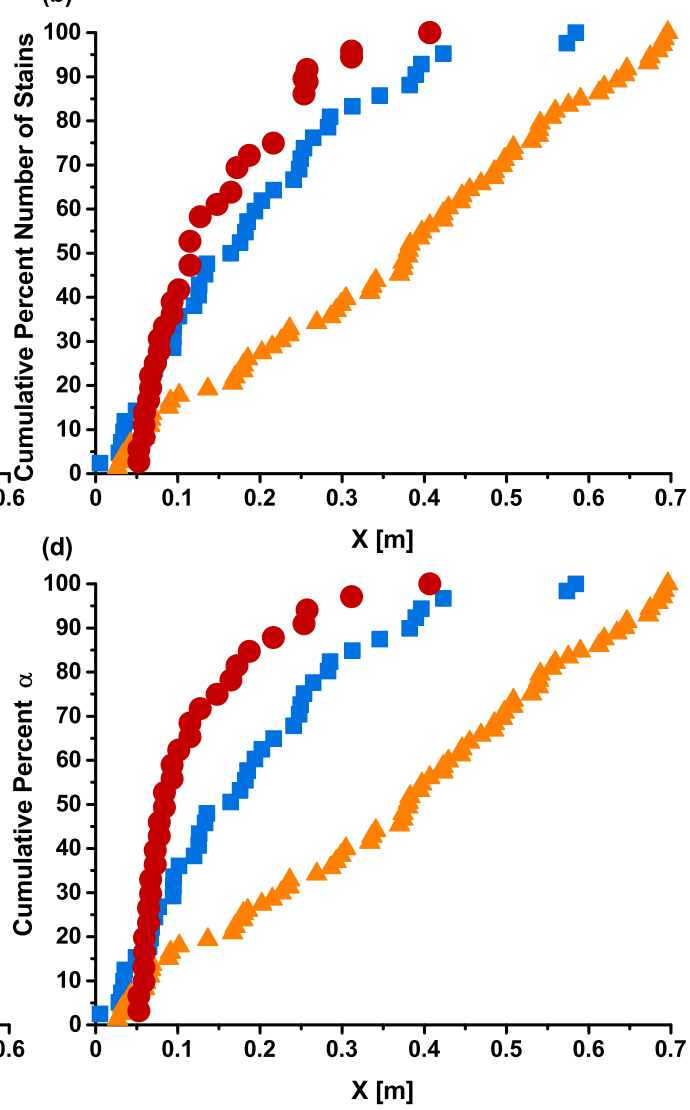

FIG. 8. Cumulative percentage for the number of stains and cumulative percentage for the impact angle. (a) and (c) Comparison of the predictions with the data sets H-a0-D300-\#1 and H-a0-D300-\#2. (b) and (d) Comparison of the predictions with the data sets H-a60-D150-\#1 and H-a60-D150-\#2. The colored symbols have the same meaning as in Figs. 6 and 7, i.e., the blue squares are for trial 1, orange triangles are for trial 2 , and large red circles are for the theoretical predictions.
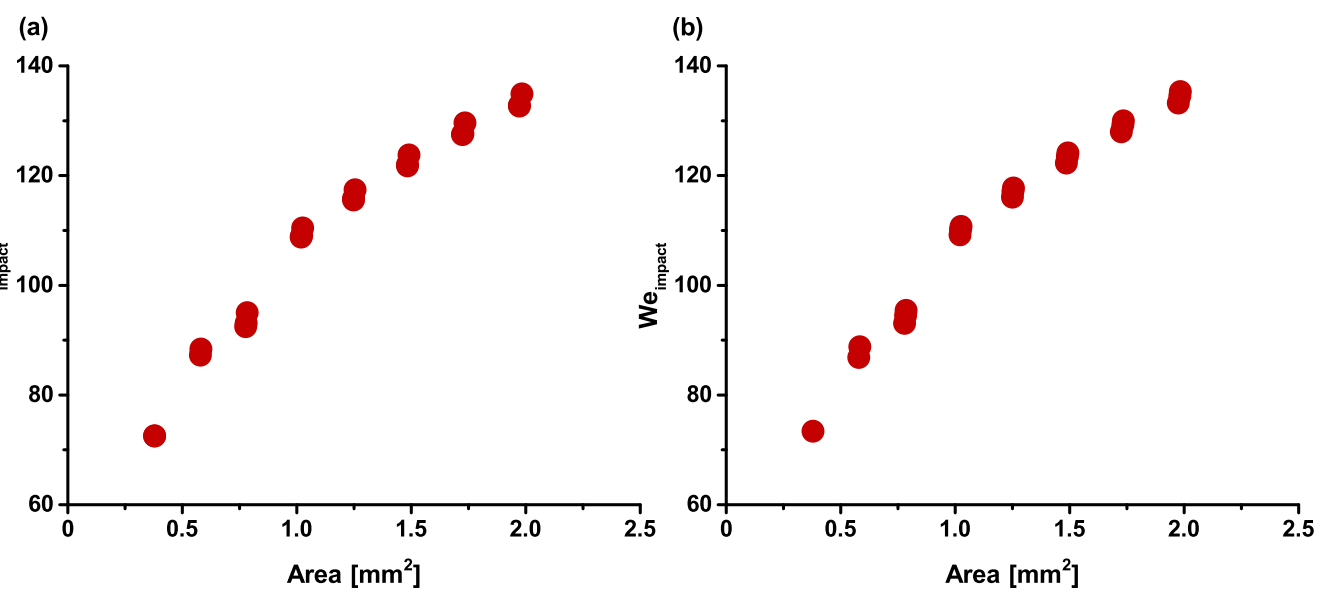

FIG. 9. Predicted impact Weber number for (a) H-a0-D300 and (b) H-a60-D150. 
(a)

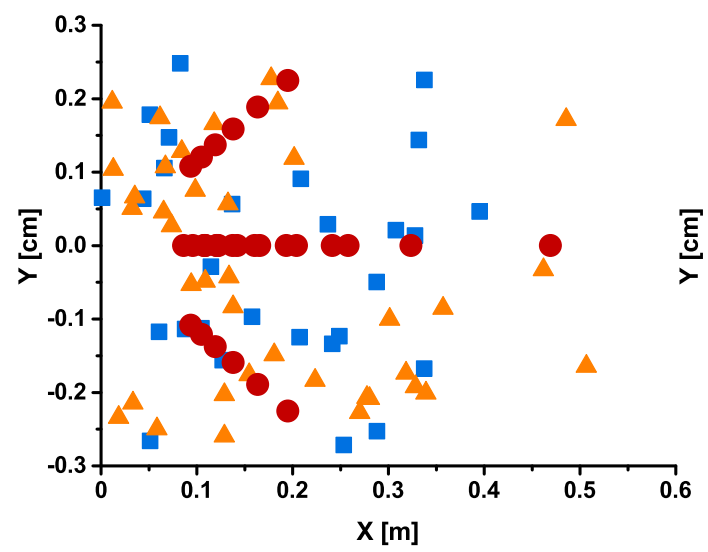

(b)

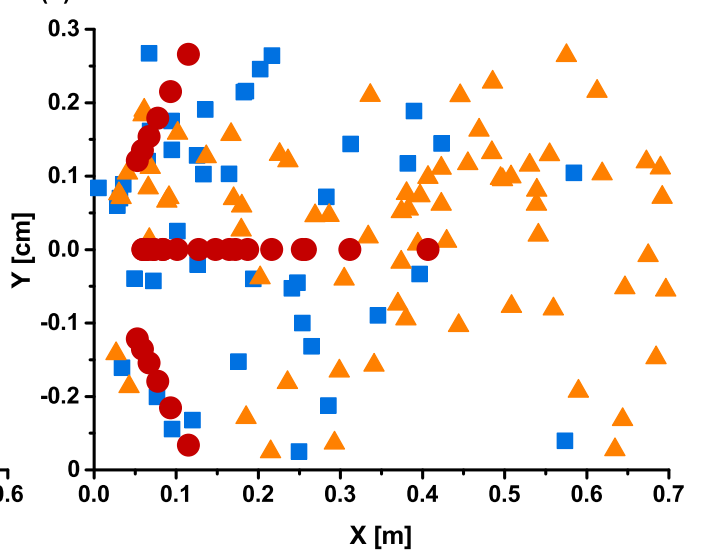

FIG. 10. Top views of predicted drop locations on the floor. (a) Predictions for the case H-a0-D300. (b) Predictions for the case H-a60-D150. The projection of the bullet path on the floor corresponds to $Y=0$. The colored symbols have the same meaning as in Figs. 6-9, i.e., the blue squares are for trial 1, orange triangles are for trial 2, and large red circles are for the theoretical predictions.

seen that for either bullet impact angle considered, the larger drops correspond to the higher impact Weber numbers.

Visualizing the stain distribution on the floor, as in Fig. 10, would provide important insight into the physical mechanisms of the back spatter. It should be emphasized that each theoretical point in Fig. 10 corresponds to a bin of drops [which might consist of one or more drops (see Sec. III)]. The theoretical predictions in Fig. 9 correspond to four generatrices of the bullet surface, $\Phi=0$, $\pi / 2, \pi$, and $3 \pi / 2$. Note that the generatrices $\Phi=0$ and $\pi$ form the uppermost and lowermost sets of red circles in Fig. 10. It should be emphasized that the numerical results underpredict the spreading distance $X$ in Fig. 10(b) for the case H-a60-D150-\#2 and agree much better for the case H-a60-D150-\#1 [similarly to the corresponding results in Figs. 8(b) and 8(d)]. A significant difference between the two experimental trials implies the effect of some additional factors, e.g., an inaccuracy in the reported bullet impact angle related to its deviation from the nominal value of $\delta=60^{\circ}$.

\section{CONCLUSION}

Experiments on back spatter of blood conducted in the present work shed some light on drop deposition on the floor. The theoretical and numerical model proposed in this work puts formation of blood drops in back spatter after a bullet impact in the framework of the Rayleigh-Taylor instability and predicts the resulting drop sizes and initial velocities in reasonable agreement with the experimental data. It should be emphasized that this theory explains the mechanism of blood drop formation in blood spatter resulting from a bullet impact and predicts the initial drop sizes and velocities. The drop cloud originating from a wound entrains a significant mass of air due to the action of viscous forces. As a result of this collective effect, air drag acting on individual drops in the cloud is significantly reduced, which is fully accounted for in the proposed model in addition to the gravity force. In calculations of the back spatter of blood this collective effect is accounted for. The theory predicted stain-size distributions, the number of stains, the average and total stain areas, cumulative percentages of the stain area and of the number of droplets, and drop impact angles and Weber numbers in certain agreement with the experimental data on back-spatter drop deposition on the floor for primary droplets with stains larger than $0.375 \mathrm{~mm}^{2}$. The cumulative percentage for the drop impact angle relative to the floor and the corresponding impact Weber number are parameters important in the framework of the analysis techniques already available to the BPA community. It should be emphasized that the prediction of the angle of blood drop impact at the surface, as well 
as of the impact Weber number by the present theory, provides the initial conditions for the impact, spreading, possible splashing [36], or penetration into the substrate [41], and drying phenomena that all affect the shape of bloodstains and their patterning associated with drying [3]. Importantly, the patterns associated with gunshots that are the topic of this work involve stains, the shape of which mainly depends on deformations during impact. As such, those blood patterns are different from blood drop patterns from sessile drops recently studied [42,43], where the shape and structure of stains were mostly caused by slow deformations associated with wetting and drying. Note also that the proposed theory of blood drop motion in air allows for a reverse calculation aimed at establishing the spatter origin based on the stain distribution on the surface. A further endeavor of the theory would be the experimental validation of the theoretical prediction of the initial velocities of blood drops in back spatter, as they are issued from the front surface of the target. The preliminary validation of this type has already been conducted by measuring such velocities from the videos available in Ref. [44]. For the videos, the measured velocities of the back-spatter blood drops at the location where they were recognizable were in the $1-1.5 \mathrm{~m} / \mathrm{s}$ range, while the predicted ones in the present work are in the $0.41-1.55 \mathrm{~m} / \mathrm{s}$ range.

\section{ACKNOWLEDGMENTS}

Support of this work by the US National Institute of Justice (Grant No. NIJ 2014-DN-BX-K036) is greatly appreciated. The authors gratefully acknowledge the skillful shooting of Christopher O'Brian for the acquisition of the experimental data, and Craig Moore who provided sponge targets.

[1] S. Weidman, Strengthening forensic science in the United States: A path forward, Committee on Identifying the Needs of the Forensic Sciences Community, National Research Council, 2009, http://www.nap.edu/catalog/12589.html.

[2] Blood-spatter analyst supports Spector defense claim, USA Today, 7/23/2007; www.usatoday30. usatoday.com/life/people/2007-07-23-phil-spector_N.htm.

[3] D. Attinger, C. Moore, A. Donaldson, A. Jafari, and H. A. Stone, Fluid dynamics topics in bloodstain pattern analysis: Comparative review and research opportunities, Forensic Sci. Int. 231, 375 (2013).

[4] V. Balthazard, R. Piedelievre, H. Desoille, and L. Derobert, XXIIe Congress de Medicine Legale de Langue Francaise, 1939; also in Ann. Med. Leg. Criminol. Police Sci. Toxicol. 19, 265 (1939).

[5] P. L. Kirk, Affidavit Regarding State of Ohio vs Samuel H. Sheppard, Court of Common Pleas, Criminal Branch, No. 64571, 26 April 1955.

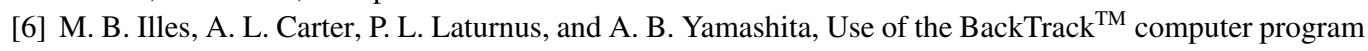
for bloodstain pattern analysis of stains from downward-moving drops, J. Can. Soc. Forensic Sci. 38, 213 (2005).

[7] A. L. Carter, The directional analysis of bloodstain patterns: Theory and experimental validation, J. Can. Soc. Forensic Sci. 34, 173 (2001).

[8] R. Kanable, BackTrack going forward, Law Enforcement Technol., August, 40 (2006).

[9] A. L. Carter, J. Forsythe-Erman, V. Hawkes, and A. B. Yamashita, Validation of the BackTrack ${ }^{\mathrm{TM}}$ suite of programs for bloodstain pattern analysis, J. Forensic Identification 56, 242 (2006).

[10] A. L. Carter, M. Illes, K. Maloney, A. B. Yamashita, B. Allen, B. Brown, L. Davidson, G. Ellis, J. Gallant, A. Gradkowski, J. Hignell, S. Jory, P. L. Laturnus, C. C. Moore, R. Pembroke, A. Richard, R. Spenard, and C. Stewart, Further validation of the BackTrack ${ }^{\mathrm{TM}}$ computer program for bloodstain pattern analysis: Precision and accuracy, Int. Assoc. Bloodstain Pattern Analysts News 21, 15 (2005).

[11] W. F. Rowe, Errors in the determination of the point of origin of bloodstains, Forensic Sci. Int. 161, 47 (2006).

[12] K. G. de Bruin, R. D. Stoel, and J. C. M. Limborgh, Improving the point of origin determination in bloodstain pattern analysis, J. Forensic Sci. 56, 1476 (2011).

[13] N. Behrooz, Bloodstain pattern analysis for determination of point of origin, B.S. thesis, University of Toronto, 2009. 
[14] B. T. Cecchetto, Nonlinear blood pattern reconstruction, MS thesis, The University of British Columbia, 2010.

[15] C. R. Varney and F. Gittes, Locating the source of projectile fluid droplets, Am. J. Phys. 79, 838 (2011).

[16] D. Attinger, C. Moore, A. Donaldson, and H. A. Stone, Fluid dynamics aspects of bloodstain pattern analysis: Comparative review and research opportunities, IABPA (International Association of Bloodstain Pattern Analysts) Training Conference, San Diego, 2013 (unpublished).

[17] N. Laan, K. G. de Bruin, D. Slenter, J. Wilhelm, M. Jermy, and D. Bonn, Bloodstain pattern analysis: Implementation of a fluid dynamic model for position determination of victims, Sci. Rep. 5, 11461 (2015).

[18] D. Poulikakos and J. Waldvogel, Heat transfer and fluid dynamics in the process of spray deposition, Adv. Heat Transfer 28, 1 (1996).

[19] N. Behrooz, L. Hulse-Smith, and S. Chandra, An evaluation of the underlying mechanisms of bloodstain pattern analysis error, J. Forensic Sci. 56, 1136 (2011).

[20] D. Denison, A. Porter, M. Mills, and R. C. Schroter, Forensic implications of respiratory derived blood spatter distributions, Forensic Sci. Int. 204, 144 (2011).

[21] M. T. Murzabayev and A. L. Yarin, Dynamics of sprinkler jets, Fluid Dyn. 20, 715 (1985).

[22] I. V. Roisman, L. Araneo, and C. Tropea, Effect of ambient pressure on penetration of a diesel spray, Int. J. Multiphase Flow 33, 904 (2007).

[23] M. Brust, C. Schaefer, R. Doerr, L. Pan, M. Garcia, P. Arratia, and C. Wagner, Rheology of Human Blood Plasma: Viscoelastic Versus Newtonian Behavior, Phys. Rev. Lett. 110, 078305 (2013).

[24] G. K. Batchelor, An Introduction to Fluid Dynamics (Cambridge University Press, Cambridge, 2002).

[25] L. G. Loitsyanskii, Mechanics of Liquids and Gases (Pergamon, Oxford, 1966).

[26] H. Lamb, Hydrodynamics (Cambridge University Press, Cambridge, 1959).

[27] G. V. Logvinovich, Hydrodynamics of Flows with Free Boundaries (Naukova Dumka, Kiev, 1969) (in Russian).

[28] A. Y. Sagomonyan, Penetration (Moscow University Publishing House, Moscow, 1974) (in Russian).

[29] H. Wagner, Über stoß- und gleitvorgänge an der oberfläche von flüssigkeiten, Z. Angew. Math. Mech. 12, 193 (1932).

[30] S. Chandrasekhar, Hydrodynamic and Hydromagnetic Stability (Dover, New York, 1981).

[31] R. Nigmatullin, Dynamics of Multiphase Systems (Hemisphere, New York,1990), Vol. 1.

[32] E. J. Lavernia, E. M. Gutirrez, J. Szekely, and N. J. Grant, A mathematical model for the liquid dynamic compaction process. Part 1: Heat flow in gas atomization, Int. J. Rapid Solid. 4, 89 (1988).

[33] A. L. Yarin, Self-similarity, in Springer Handbook of Experimental Fluid Mechanics, edited by C. Tropea, A. L. Yarin, and J. F. Foss (Springer, Heidelberg, 2007), pp. 57-82.

[34] P. B. S. Lissaman and C. A. Shollenberger, Formation flight of birds, Science 168, 1003 (1970).

[35] B. L. Scheller and D. W. Bousfield, Newtonian drop impact with a solid surface, AIChE J. 41, 1357 (1995).

[36] A. L. Yarin, Drop impact dynamics: Splashing, spreading, receding, bouncing, Annu. Rev. Fluid Mech. 38, 159 (2006).

[37] C. Antonini, A. Amirfazli, and M. Marengo, Drop impact and wettability: From hydrophilic to superhydrophobic surfaces, Phys. Fluids 24, 102104 (2012).

[38] C. D. Adam, Fundamental studies of bloodstain formation and characteristics, Forensic Sci. Int. 219, 76 (2012).

[39] M. C. Taylor, T. L. Laber, B. P. Epstein, D. S. Zamzow, and D. P. Baldwin, The effect of firearm muzzle gases on the backspatter of blood, Int. J. Legal Med. 125, 617 (2010).

[40] C. Rizer, Police Mathematics (Thomas, Springfield, 1955).

[41] R. P. Sahu, S. Sett, A. L. Yarin, and B. Pourdeyhimi, Impact of aqueous suspension drops onto non-wettable membranes: Hydrodynamic focusing and penetration of nanoparticles, Colloids Surf. A 467, 31 (2015).

[42] D. Brutin, B. Sobac, B. Loquet, and J. Sampol, Pattern formation in drying drops of blood, J. Fluid Mech. 667, 85 (2011).

[43] R. Chen, L. Zhang, S. Zang, and W. Shen, Blood drop patterns: Formation and applications, Adv. Colloid Interface Sci. 231, 1 (2016).

[44] Ames Laboratory, Iowa State University, www.ameslab.gov/mfrc/bpa-videos. 